\title{
Stability of DC micro-grid for urban railway systems
}

\author{
Sarah Nasr ${ }^{\mathrm{a}, \mathrm{b}}$, Marc Petit ${ }^{\mathrm{a}}$, Marius Iordache ${ }^{\mathrm{b}}$, Olivier Langlois ${ }^{\mathrm{b}^{*}}$ \\ ${ }^{a}$ GeePs $\mid$ Group of electrical engineering - Paris UMR CNRS 8507, CentraleSupélec, Univ Paris-Sud, Sorbonne Universités, \\ UPMC Univ Paris 06, 3-11 rue Joliot-Curie, Plateau de Moulon F-91192 Gif-sur-Yvette CEDEX \\ ${ }^{b}$ Alstom Transport SA, 48 rue Albert Dhalenne, Saint-Ouen 93400, France
}

\begin{abstract}
This paper studies the stability of a DC Micro-grid integrated in urban railway systems in order to recover trains braking energy. It is a green solution based on storing the excess of braking energy in a hybrid storage system and reusing it in non-railway applications such as auxiliary loads in a station or electric vehicles in proximity, which will increase the global energy efficiency. The risk of instability caused by constant power loads is detailed and solved using backstepping approach. It is shown that this problem can be solved by controlling the energy storage system.
\end{abstract}

Keywords: DC Micro-grid, stability, backstepping, railway, hybrid storage system

\section{Introduction}

In DC railway systems, there is a possibility to regenerate braking energy and to exchange it between trains. But in case of classic traction power supply consisting of diode-rectifiers with unidirectional power flow, there is no energy exchange with surrounding energy network and no possibility to integrate smart energy management system. Smart railway electrification will provide energy savings by accommodating all distributed generations (braking trains, renewable energy...) and storage systems (batteries, supercapacitors...). It will dynamically optimize the total power consumption and enhance power quality and system's efficiency. Railway system will no longer be a passive load consuming energy from the grid. It will take part of a larger smart grid and communicate with "non-railway" applications such as smart buildings, electrical vehicle charging stations... In this paper, the concept of recovering trains braking energy through a DC Micro-grid [1] is first presented. The stability of the system is then highlighted. In fact, when multi-sources and constant power loads (CPL) are connected to a DC Busbar, stability issues are faced. In the literature, this problem is usually analysed by small signal stability study which linearize the system around an operating point and makes it possible to use linear tools such as Bode and Nyquist diagrams, Routh-Hurwitz criterion [2]-[4]. However, railway braking energy is in the shape of unpredictable power peaks that can go up to $3 \mathrm{MW}$ in few seconds which make it not possible to define only one operating point to the system. Therefore, it is necessary to study the Micro-grid in its non-linear form. On the other hand, some have chosen damping filters with passive elements [3], [5]. This solution will increase the cost by over-sizing components (resistors, capacitors) which will also increase losses. Therefore, the backstepping approach [6] will be detailed. It will act on the control of the hybrid storage system and more precisely on the supercapacitor. It will stabilize the Micro-grid taking into consideration the dynamic evolution of the system.

\section{Railway DC Micro-Grid}

Nowadays, one of the challenges in railway systems is recovering the braking energy and avoiding

\footnotetext{
* Manuscript received April 30, 2015; revised August 11, 2015.

Sarah NASR. Tel.: +33-1-57061275; E-mail address: sarah.nasr@transport.alstom.com.

doi: $10.12720 /$ sgce.4.3.261-268
} 
losses. In fact, when an electric train brakes, it converts the mechanical kinetic energy to electrical energy and feeds it back into the source line (catenary or 3rd rail). This causes voltage increase at pantograph level. If another train is accelerating closely enough, the energy sent back to the catenary will be consumed by this train. In the opposite case, regenerated energy causes overvoltage which can damage the infrastructure. Therefore, when the voltage exceeds a threshold (e.g. $900 \mathrm{~V}$ for $750 \mathrm{~V}$ electrification system), the energy is burned in rheostats embedded in trains. The solution is to recover this energy before stimulating the rheostats through different technologies such as inverters, storage... The Railway DC Micro-grid, allows re-using the braking energy internally by the same operator but in different electrical applications, for example, lighting and escalators inside a metro station, electrical vehicles and buses parked outside the station... This eco-friendly solution will permit reducing energy losses, saving energy and thereby decreasing the total energy bill. In this paper, we will consider a scenario where the braking energy is used to charge electrical hybrid buses parked outside a metro station, thus enhancing the green multi-modal public transport deployment and improving the asset management of an urban transportation system.

\subsection{Concept}

Fig. 1(a) represents the global architecture of the DC Micro-grid. It consists of the following components:

- 900V DC busbar

- Two-level bidirectional inverter (4) connected to the LV power supply already available in the metro station

- DC/DC converters connecting the energy storage (2), railway system (1) and the hybrid buses (3) to the common DC busbar

- Hybrid energy storage device (5) containing supercapacitors (SC) and batteries.

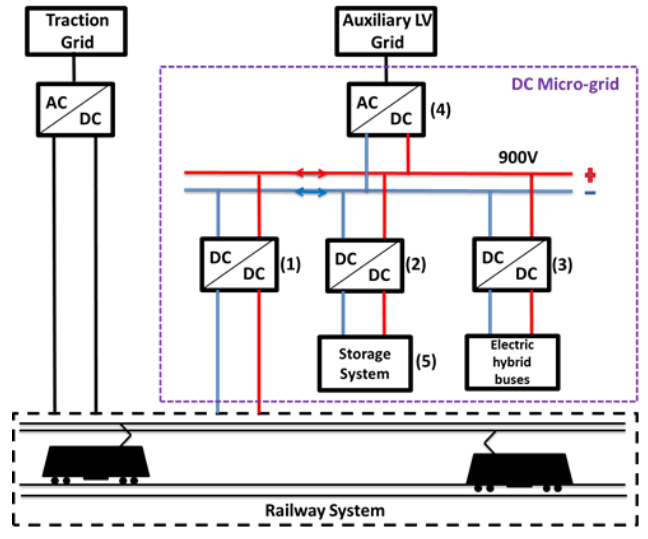

(a)

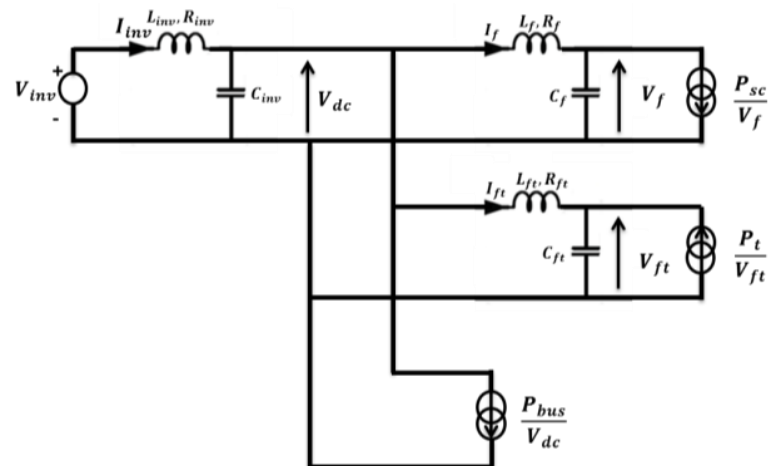

(b)

Fig. 1. The DC Micro-grid architecture: (a) global model and (b) simplified model.

The DC Micro-grid is connected to auxiliary low voltage grid. Therefore, a connection between both auxiliary and traction grids, usually separated in the case of diode rectifier substations, is possible through the DC Micro-grid. When a train brakes, the catenary (or third rail) voltage increases. Once the voltage exceeds a specific threshold, the converter (1) will recover the braking energy and inject it into the DC bus. This converter should be regulated to give priority to energy exchanging between trains. The second DC/DC converter (2) will store the energy and help reducing the DC busbar voltage. The AC/DC bidirectional inverter is used to regulate the DC bus voltage to avoid voltage peaks or drops.

\subsection{Instability risk}

In this use case, trains' braking energy is stored and then used to charge the electric hybrid buses. 
These buses consume a constant power of $200 \mathrm{~kW}$ during 4-5 minutes. The problem of connecting a CPL is that its linear model behaves as a negative resistance (Fig. 2). It will then cause instability because it amplifies filters' dynamics in a resonant circuit. In the opposite case of Constant Power Source (CPS), its linear model behaves as positive resistance. It will damp the system dynamics caused by the input filter. The figures below show the behaviour of CPL and CPS [6].

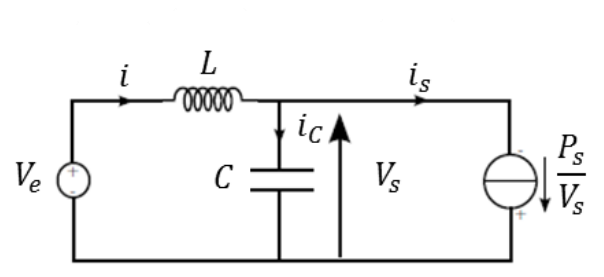

(a)

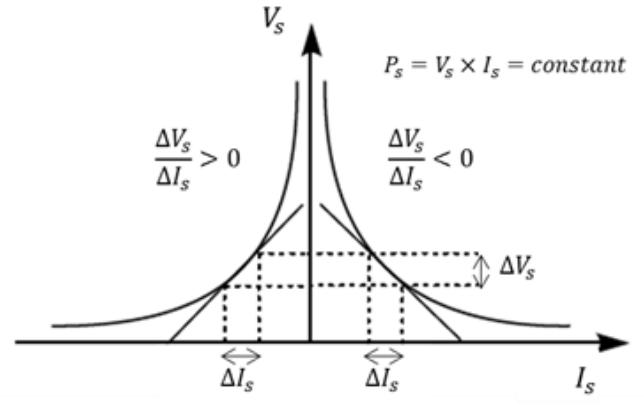

(b)

Fig. 2. Linear model of a constant power load: (a) DC electrical network with CPL/CPS and (b) negative and positive impedance of CPL/CPS [6].

\section{Backstepping Approach}

\subsection{Micro-grid modelling}

In order to simplify the problem, the converters are represented by current sources operating in consumption/generation modes. In figure 1.b, the studied system is presented where $V_{i n v}$ represents the inverter's output voltage $\left(V_{i n v}=900 \mathrm{~V}\right), P_{s c}$ the power absorbed by the hybrid storage system, $P_{t}$ the braking power recovered by the trains and $P_{b u s}$ the constant power absorbed by the electric hybrid bus. The set of equations representing the system is given below. Note that $V_{i n v}$ and $P_{b u s}$ are constant, $P_{t}$ is also considered as a constant because the system dynamics are much faster than the braking power variation. $P_{s c}$ is the control command of the storage.

$$
\left\{\begin{array} { l } 
{ L _ { i n v } \frac { d I _ { i n v } } { d t } = V _ { i n v } - V _ { d c } - R _ { i n v } . I _ { i n v } } \\
{ L _ { f } \frac { d I _ { f } } { d t } = V _ { d c } - V _ { f } - R _ { f } \cdot I _ { f } } \\
{ L _ { f t } \frac { d I _ { f t } } { d t } = V _ { d c } - V _ { f t } - R _ { f t } . I _ { f t } }
\end{array} \left\{\begin{array}{l}
C_{i n v} \frac{d V_{d c}}{d t}=I_{i n v}-I_{f}-. I_{f t}-P_{b u s} / V_{d c} \\
C_{f} \frac{d V_{f}}{d t}=I_{f}-P_{s c} / V_{f} \\
C_{f t} \frac{d V_{f t}}{d t}=I_{f t}+P_{t} / V_{f t}
\end{array}\right.\right.
$$

Considering the vector $\mathrm{X}_{0}=\left[\mathrm{I}_{\mathrm{inv} 0}, \mathrm{I}_{\mathrm{f} 0}, \mathrm{I}_{\mathrm{ft} 0}, \mathrm{~V}_{\mathrm{dc} 0}, \mathrm{~V}_{\mathrm{f} 0}, \mathrm{~V}_{\mathrm{ft} 0}\right]$ as an equilibrium point, equations (1) can be centred round $\mathrm{X}_{0}$ with the following variable changes:

$$
\left\{\begin{array}{l}
x_{1}=I_{i n v}-I_{i n v 0} \\
x_{2}=I_{f}-I_{f 0} \\
x_{3}=I_{f t}-I_{f t 0}
\end{array}\right.
$$$$
\left\{\begin{array}{l}
x_{4}=V_{d c}-V_{d c 0} \\
x_{5}=V_{f}-V_{f 0} \\
x_{6}=V_{f t}-V_{f t 0}
\end{array}\right.
$$

After replacing (2) in (1), the new equivalent system is given in (3) where the equilibrium point $\mathrm{X}_{0}$ is the system's origin: 


$$
\left\{\begin{array} { l } 
{ L _ { i n v } \dot { x } _ { 1 } = - R _ { i n v } x _ { 1 } - x _ { 4 } } \\
{ L _ { f } \dot { x } _ { 2 } = - R _ { f } x _ { 2 } + x _ { 4 } - x _ { 5 } } \\
{ L _ { f t } \dot { x } _ { 3 } = - R _ { f t } x _ { 3 } + x _ { 4 } - x _ { 6 } }
\end{array} \left\{\begin{array}{l}
C_{i n v} \dot{x}_{4}=x_{1}-x_{2}-x_{3}+\frac{P_{b u s} \cdot x_{4}}{\left(x_{4}+V_{d c 0}\right) \cdot V_{d c 0}} \\
C_{f} \dot{x}_{5}=x_{2}+\frac{P_{s c} \cdot x_{5}}{\left(x_{5}+V_{f 0}\right) \cdot V_{f 0}} \\
C_{f t} \dot{x}_{6}=x_{3}-\frac{P_{t} \cdot x_{6}}{\left(x_{6}+V_{f t 0}\right) \cdot V_{f t 0}}
\end{array}\right.\right.
$$

\subsection{System stabilization}

After modelling the DC Micro-grid and centring the equations round the equilibrium point $\mathrm{X}_{0}$, the backstepping control technique requires that equations describing the dynamics of the system (3) are presented in cascaded form. To achieve that, a change of variables is done as follows:

$$
\left[\begin{array}{c}
z_{1} \\
z_{2} \\
z_{3} \\
z_{4} \\
z_{5} \\
z_{6}
\end{array}\right]=\left[\begin{array}{c}
L_{i n v} x_{1}+L_{f t} x_{3} \\
C_{f t} x_{6} \\
L_{i n v} x_{1}-L_{f t} x_{3} \\
C_{i n v} x_{4} \\
L_{f} x_{2} \\
C_{f} x_{5}
\end{array}\right] \text { and }\left[\begin{array}{c}
z_{20} \\
z_{40} \\
z_{60}
\end{array}\right]=\left[\begin{array}{c}
C_{f t} V_{f t 0} \\
C_{i n v} V_{d c 0} \\
C_{f} V_{f 0}
\end{array}\right] \text {, with } \frac{R_{f t}}{L_{f t}}=\frac{R_{i n v}}{L_{i n v}}
$$

After applying (4), equations (3) can be expressed in the following cascaded form where ' $u$ ' is the backstepping command that will be added to the classic command $\mathrm{P}_{\mathrm{sc} 0}$ to ensure a global asymptotic stability:

$$
\left\{\begin{array}{l}
\dot{z}_{1}=\varphi_{1}\left(z_{1}\right)+\psi_{1} z_{2} \\
\dot{z}_{2}=\varphi_{2}\left(z_{1}, z_{2}\right)+\psi_{2} z_{3} \\
\dot{z}_{3}=\varphi_{3}\left(z_{1}, z_{2}, z_{3}\right)+\psi_{3} z_{4}
\end{array}\right.
$$$$
\left\{\begin{array}{l}
\dot{z}_{4}=\varphi_{4}\left(z_{1}, z_{2}, z_{3}, z_{4}\right)+\psi_{4} z_{5} \\
\dot{z}_{5}=\varphi_{5}\left(z_{1}, z_{2}, z_{3}, z_{4}, z_{5}\right)+\psi_{5} z_{6} \\
\dot{z}_{6}=\varphi_{6}\left(z_{1}, z_{2}, z_{3}, z_{4}, z_{5}, z_{6}\right)+\psi_{6} u
\end{array}\right.
$$

with,

$$
\begin{cases}\varphi_{1}\left(z_{1}\right)=-R_{i n v} \cdot z_{1} / L_{i n v} & \psi_{1}=-\frac{1}{C_{f t}} \\ \varphi_{2}\left(z_{1}, z_{2}\right)=\frac{z_{1}}{2 L_{f t}}-\frac{C_{f t} P_{t} z_{2}}{z_{20}\left(z_{20}+z_{2}\right)} & \psi_{2}=-\frac{1}{2 L_{f t}} \\ \varphi_{3}\left(z_{1}, z_{2}, z_{3}\right)=-\frac{R_{i n v}}{2 L_{i n v}}\left(z_{3}+z_{1}\right)-\frac{R_{f t}}{2 L_{f t}}\left(z_{3}-z_{1}\right)+\frac{z_{2}}{C_{f t}} & \psi_{3}=-\frac{2}{C_{i n v}} \\ \varphi_{4}\left(z_{1}, z_{2}, z_{3}, z_{4}\right)=\frac{1}{2 L_{i n v}}\left(z_{3}+z_{1}\right)+\frac{1}{2 L_{f t}}\left(z_{3}-z_{1}\right)+\frac{C_{i n v} P_{b u s} z_{4}}{z_{40}\left(z_{40}+z_{4}\right)} & \psi_{4}=-\frac{1}{L_{f}} \\ \varphi_{5}\left(z_{1}, z_{2}, z_{3}, z_{4}, z_{5}\right)=-\frac{R_{f}}{L_{f}} z_{5}+\frac{1}{C_{i n v}} z_{4} & \psi_{5}=-\frac{1}{C_{f}} \\ \varphi_{6}\left(z_{1}, z_{2}, z_{3}, z_{4}, z_{5}, z_{6}\right)=\frac{1}{L f} z_{5}+\frac{C_{f} P_{s c 0} z_{6}}{z_{60}\left(z_{60}+z_{6}\right)} & \psi_{6}=-\frac{C_{f}}{\left(z_{60}+z_{6}\right)}\end{cases}
$$

where $R_{i n v}, L_{i n v}, R_{f}, L_{f}, R_{f t}, L_{f t}, C_{i n v}, C_{f}$ and $C_{f t}$ are constants characterizing the system; $P_{b u s}$ and $P_{t}$ are 
measurable, $P_{s c 0}$ is the classic command of the storage system and it is equal to: $P_{s c 0}=P_{t}-P_{b u s}$. The state vector presentation (5) will allow calculating the command ' $\mathrm{u}$ ' by dividing the system to six cascaded subsystems $\mathrm{S}_{\mathrm{i}}$ defined by the state vector $\left[z_{1} \ldots z_{i}\right]$. The backstepping method consists of calculating a positive-definite Lyapunov function $V_{i}$ for each subsystem $\mathrm{S}_{\mathrm{i}}$. The global stability is then achieved by stabilizing each subsystem. The subsystem $\mathrm{S}_{6}$ corresponds to the full system. Detailed calculus will be done for the first two subsystems; the rest can be done in the same manner. The command value of each $S_{\mathrm{i}}$ will be then directly given.

- Stabilization of subsystem $S_{1}: \dot{z}_{1}=\varphi_{1}\left(z_{1}\right)+\psi_{1} z_{2}$

For $\mathrm{S}_{1}, z_{2}$ represent the input command of the subsystem. The command value $z_{1}^{*}$ of $z_{1}$ is considered equal to zero. The state error $\varepsilon_{1}$ is given by:

$$
\varepsilon_{1}=z_{1}-z_{1}^{*} \Rightarrow \dot{\varepsilon}_{1}=\dot{z}_{1}-\dot{z}_{1}^{*}=\varphi_{1}\left(z_{1}\right)+\psi_{1} z_{2}-\dot{z}_{1}^{*}
$$

We consider the positive-definite Lyapunov function:

$$
V_{1}=\frac{1}{2 L_{f t}} \varepsilon_{1}^{2} \Rightarrow \dot{V}_{1}=\frac{1}{L_{f t}} \dot{\varepsilon}_{1} \varepsilon_{1}=\frac{1}{L_{f t}} \varepsilon_{1}\left(\varphi_{1}\left(z_{1}\right)+\psi_{1} z_{2}-\dot{z}_{1}^{*}\right)
$$

$\mathrm{S}_{1}$ is asymptotically stable if the derivation of $V_{1}$ is negative-definite. This condition is fulfilled by the following equation:

$$
\varphi_{1}\left(z_{1}\right)+\psi_{1} z_{2}-\dot{z}_{1}^{*}=-k_{1} e_{1} \Rightarrow \dot{V}_{1}=-k_{1} e_{1}^{2}<0
$$

where $k_{1}>0$ is a parameter to be defined depending on the dynamic behaviour of S1.

In order to insure convergence to $\mathrm{X}_{0}$, the command value $z_{2}^{*}$ of $z_{2}$ should be equal to:

$$
z_{2}^{*}=\frac{1}{\psi_{1}}\left(-\varphi_{1}\left(z_{1}\right)-k_{1} e_{1}+\dot{z}_{1}^{*}\right)
$$

\section{- Stabilization of subsystem $\mathrm{S}_{2}: \quad\left\{\dot{z}_{1}, \dot{z}_{2}\right\}$}

For $\mathrm{S}_{2}, z_{3}$ is the input command that will let the output $z_{2}$ equal to $z_{2}^{*}$. The state error vector is then:

$$
\varepsilon_{2}=z_{2}-z_{2}^{*} \Rightarrow \dot{\varepsilon}_{2}=\dot{z}_{2}-\dot{z}_{2}^{*}=\varphi_{2}\left(z_{1}, z_{2}\right)+\psi_{2} z_{3}-\dot{z}_{2}^{*}
$$

We consider the positive-definite Lyapunov function:

$$
\begin{aligned}
& V_{2}=\frac{1}{2 C_{f t}} \varepsilon_{2}^{2}+V_{1} \Rightarrow \\
& \dot{V}_{2}=\dot{V}_{1}+\frac{1}{C_{f t}} \dot{\varepsilon}_{2} \varepsilon_{2}=\frac{1}{L_{f t}} \varepsilon_{1}\left(\varphi_{1}\left(z_{1}\right)+\psi_{1}\left(\varepsilon_{2}+\dot{z}_{2}^{*}\right)-\dot{z}_{1}^{*}\right)+\frac{1}{C_{f t}} \varepsilon_{2}\left(\varphi_{2}\left(z_{1}, z_{2}\right)+\psi_{2} z_{3}-\dot{z}_{2}^{*}\right)
\end{aligned}
$$

$\mathrm{S}_{2}$ is asymptotically stable if the derivation of $V_{2}$ is negative-definite. This condition is fulfilled by the following equation:

$$
\frac{C_{f t}}{L_{f t}} \psi_{1} \varepsilon_{1}+\varphi_{2}\left(z_{1}, z_{2}\right)+\psi_{2} z_{3}-\dot{z}_{2}^{*}=-k_{2} \varepsilon_{2} \text { with } k_{2}>0
$$


The input command of $S_{2}$ is then:

$$
z_{3}^{*}=\frac{1}{\psi_{2}}\left(-\frac{C_{f t}}{L_{f t}} \psi_{1} \varepsilon_{1}-\varphi_{2}\left(z_{1}, z_{2}\right)+\dot{z}_{2}^{*}-k_{2} \varepsilon_{2}\right)
$$

- Stabilization of subsystem $\mathrm{S}_{3}:\left\{\dot{z}_{1}, \dot{z}_{2}, \dot{z}_{3}\right\}$

As for the previous subsystems, the input command $z_{4}^{*}$ that will insure stability of $S_{3}$ is:

$z_{4}^{*}=\frac{1}{\psi_{3}}\left(-\frac{L_{f t}}{C_{f t}} \psi_{2} \varepsilon_{2}-\varphi_{3}\left(z_{1}, z_{2}, z_{3}\right)+\dot{z}_{3}^{*}-k_{3} \varepsilon_{3}\right)$ with $k_{3}>0$

- Stabilization of subsystem $\mathrm{S}_{4}:\left\{\dot{z}_{1}, \dot{z}_{2}, \dot{z}_{3}, \dot{z}_{4}\right\}$

The input command $z_{5}^{*}$ that will insure stability of $S_{4}$ is:

$z_{5}^{*}=\frac{1}{\psi_{4}}\left(-\frac{C_{i n v}}{L_{f t}} \psi_{3} \varepsilon_{3}-\varphi_{4}\left(z_{1}, z_{2}, z_{3}, z_{4}\right)+\dot{z}_{4}^{*}-k_{4} \varepsilon_{4}\right)$ with $k_{4}>0$

- Stabilization of subsystem $\mathrm{S}_{5}:\left\{\dot{z}_{1}, \dot{z}_{2}, \dot{z}_{3}, \dot{z}_{4}, \dot{z}_{5}\right\}$

The input command $z_{6}^{*}$ that will insure stability of $S_{4}$ is:

$z_{6}^{*}=\frac{1}{\psi_{5}}\left(-\frac{L_{f}}{C_{i n v}} \psi_{4} \varepsilon_{4}-\varphi_{5}\left(z_{1}, z_{2}, z_{3}, z_{4}, z_{5}\right)+\dot{z}_{5}^{*}-k_{5} \varepsilon_{5}\right)$ with $k_{5}>0$

- Stabilization of subsystem $\mathrm{S}_{6}:\left\{\dot{z}_{1}, \dot{z}_{2}, \dot{z}_{3}, \dot{z}_{4}, \dot{z}_{5}, \dot{z}_{6}\right\}$

$\mathrm{S}_{6}$ represents the Micro-grid. The input command ' $u$ ' that will insure the global stability of the system is:

$u=\frac{1}{\psi_{6}}\left(-\frac{C_{f}}{L_{f}} \psi_{5} \varepsilon_{5}-\varphi_{6}\left(z_{1}, z_{2}, z_{3}, z_{4}, z_{5}, z_{6}\right)+\dot{z}_{6}^{*}-k_{6} \varepsilon_{6}\right)$ with $k_{6}>0$

The Lyapunov function $V$ of the system is:

$V=\frac{1}{2 L_{f t}} \varepsilon_{1}^{2}+\frac{1}{2 C_{f t}} \varepsilon_{2}^{2}+\frac{1}{2 L_{f t}} \varepsilon_{3}^{2}+\frac{1}{2 C_{i n v}} \varepsilon_{4}^{2}+\frac{1}{2 L_{f}} \varepsilon_{5}^{2}+\frac{1}{2 C_{f}} \varepsilon_{6}^{2}>0$

The derivative of $V$ is:

$$
\dot{V}=-\frac{k_{1}}{L_{f t}} \varepsilon_{1}^{2}-\frac{k_{2}}{C_{f t}} \varepsilon_{2}^{2}-\frac{k_{3}}{L_{f t}} \varepsilon_{3}^{2}-\frac{k_{4}}{C_{i n v}} \varepsilon_{4}^{2}-\frac{k_{5}}{L_{f}} \varepsilon_{5}^{2}-\frac{k_{6}}{C_{f}} \varepsilon_{6}^{2}<0
$$

(16) and (17) ensures the asymptotic stability of the system around the origin $\mathrm{X}_{0}$ which is calculated dynamically using Newton-Raphson method.

\section{Simulation and Results}

The simplified model of the DC Micro-grid shown in Fig. 1(b) is simulated using Matlab-Simulink. 
The control command (15) is applied to the storage system. The $k_{i}$ parameters used are:

$$
k_{1}=50, k_{2}=30, k_{3}=13, k_{4}=8, k_{5}=300, k_{6}=10^{-5} .
$$

The values of the electrical components of the DC Micro-grid are: $C_{f}=0,041 \mathrm{~F}, C_{f i}=0,011 \mathrm{~F}$, $C_{i n v}=3.532 .10^{-4} \mathrm{~F}, L_{f}=10.12 \mu \mathrm{H}, L_{f t}=9.45 \mu \mathrm{H}, L_{i n v}=11.9 \mu \mathrm{H}, R_{f}=0.25 \mathrm{~m} \Omega, R_{f i}=0.23 \mathrm{~m} \Omega, R_{i n v}=0.29 \mathrm{~m} \Omega$.

Fig. 3 shows profiles of the recovered braking power and the electric hybrid bus charging power. The first one increases from zero up to $1 \mathrm{MW}$. The second one is $200 \mathrm{~kW}$ constant power.

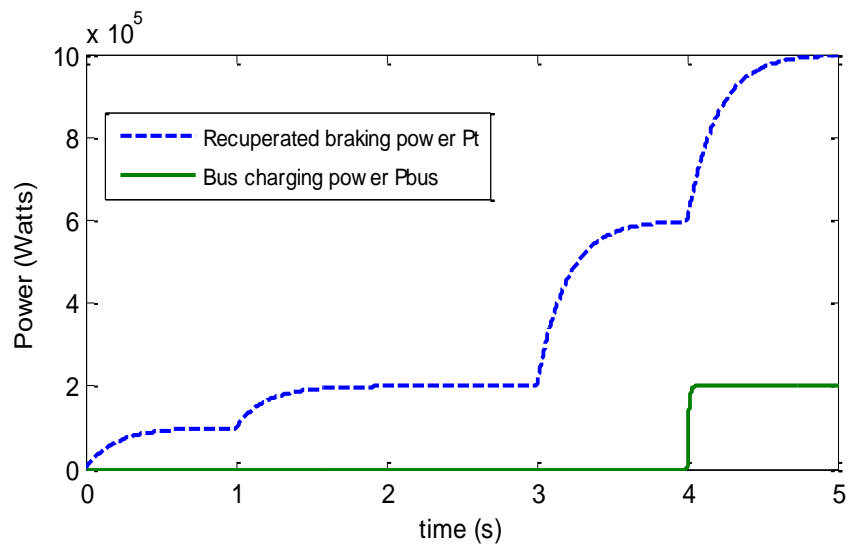

Fig. 3. Recovered power and bus charging power simulated profile.
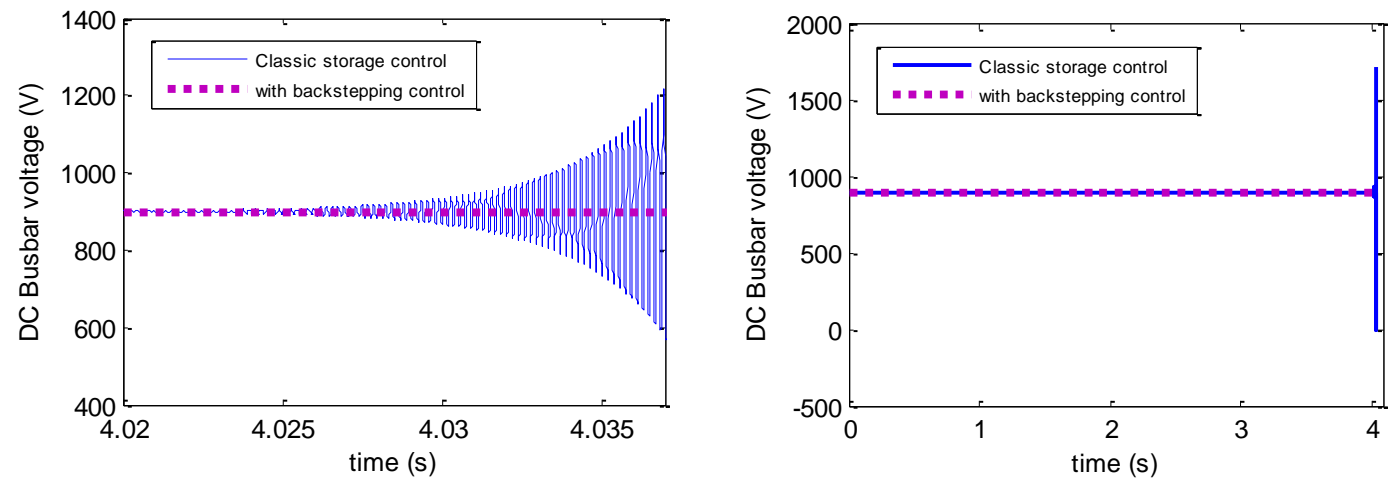

Fig. 4. DC busbar voltages for classic and backstepping controls.

Fig. 4 compares between the classic storage control $\left(P_{s c 0}=P_{t}-P_{b u s}\right)$ and the one with backstepping control $\left(P_{s c}=P_{s c 0}+u\right)$. Note that the simulation with the classic control stops at time $=4,035 \mathrm{~s}$ for divergence reason. The backstepping control allowed stabilizing the system and by that the DC busbar voltage $\left(V_{d c} \cong 900 \mathrm{~V}\right)$.

\section{Conclusion}

In this paper, the concept of DC Micro-grid for urban railway systems was first presented. Its main role is to recover trains braking power, store it in a hybrid storage system and use this energy to charge electric hybrid buses with a constant power. The problem of instability that can be caused by a constant power load was then explained. A solution called backstepping approach was detailed and a comparison of simulation results between classic and backstepping controls proved that the backstepping control is capable of converging and stabilizing a low-damped system. The next step will be simulating 
backstepping control on the complete DC Micro-grid with all the converters shown in Fig. 1(a).

\section{References}

[1] Nasr S, Iordache M, Petit M. Smart micro-grid integration in DC railway systems. Presented at: Innovative Smart Grid Technologies Conference Europe, 2014.

[2] Middlebrook RD. Input filter considerations in design and application of switching regulators. In: Proc. IAS'76, 1976:366-382.

[3] Godoy E, Coll. Regulation Industrielle. Paris: Dunod, 2007.

[4] Barruel F, Retiere N, Schanen JL, Caisley A. Stability approach for vehicles dc power network: application to aircraft onboard system. In: Proc. IEEE 36th Power Electronics Specialists Conference, June 2005:1163-1169.

[5] Erickson R. Optimal single resistors damping of input filters. In: Proc. Applied Power Electronics Conference and Exposition, Fourteenth Annual IEEE, Mar. 1999; 2:1073-1079.

[6] Hamache D, Fayaz A, Godoy E, Karimi C, Stabilization of a DC electrical network via backstepping approach. Presented at: $23^{\text {rd }}$ International Symposium on Industrial Electronics, 2014. 